\title{
Employing electro-mechanical analogies for co-resonantly coupled cantilever sensors
}

\author{
Julia Körner ${ }^{1}$, Christopher F. Reiche ${ }^{1}$, Bernd Büchner ${ }^{1,2}$, Thomas Mühl ${ }^{1}$, and Gerald Gerlach ${ }^{3}$ \\ ${ }^{1}$ Leibniz Institute for Solid State and Materials Research IFW Dresden, Helmholtzstr. 20, \\ 01069 Dresden, Germany \\ ${ }^{2}$ Institut für Festkörperphysik, Technische Universität Dresden, 01062 Dresden, Germany \\ ${ }^{3}$ Institut für Festkörperelektronik, Technische Universität Dresden, 01062 Dresden, Germany
}

Correspondence to: Thomas Mühl (t.muehl@ifw-dresden.de)

Received: 3 March 2016 - Revised: 15 June 2016 - Accepted: 16 June 2016 - Published: 13 July 2016

\begin{abstract}
Understanding the behaviour of mechanical systems can be facilitated and improved by employing electro-mechanical analogies. These analogies enable the use of network analysis tools as well as purely analytical treatment of the mechanical system translated into an electric circuit. Recently, we developed a novel kind of sensor set-up based on two coupled cantilever beams with matched resonance frequencies (co-resonant coupling) and possible applications in magnetic force microscopy and cantilever magnetometry. In order to analyse the sensor's behaviour in detail, we describe it as an electric circuit model. Starting from a simplified coupled harmonic oscillator model with neglected damping, we gradually increase the complexity of the system by adding damping and interaction elements. For each stage, various features of the coupled system are discussed and compared to measured data obtained with a co-resonant sensor. Furthermore, we show that the circuit model can be used to derive sensor parameters which are essential for the evaluation of measured data. Finally, the much more complex circuit representation of a bending beam is discussed, revealing that the simplified circuit model of a coupled harmonic oscillator is a very good representation of the sensor system.
\end{abstract}

\section{Introduction}

Electro-mechanical analogies are a suitable tool to describe, understand and analyse the behaviour of mechanical systems (Firestone, 1933). Developed at the end of the 19th century (Hähnle, 1931), two conventions have been introduced in order to model the mechanical system as an electrical circuit. One analogy is based on the principle of cause and effect (Klotter, 1950), while the other analogy makes use of the equivalence of the system's differential equations. We will use the latter one to describe the behaviour of a sensor consisting of two coupled oscillating systems made of cantilever beams because it has been shown that it is a suitable analogy for these kinds of systems (Hähnle, 1931).

In the following, we will start with a short description of the mechanical system which is translated into an electric circuit model. From there we analyse the system's behaviour for different cases, i.e. without and with damping, and then show a possible way to derive useful information to characterize the sensor.

\section{The sensor concept}

Cantilever-based measurement techniques are widely spread, for example for the determination of magnetic sample properties as in magnetic force microscopy or cantilever magnetometry (Gysin et al., 2011). They can furthermore be employed as mass sensors (Sökmen et al., 2010). The measurement signal is obtained by either measuring the static deflection or the shift of the oscillation parameters, e.g. the resonance frequency, of the cantilever. In all these cases the sensitivity of the technique is mainly influenced by the cantilever's spring constant, which is closely related to its spatial dimensions. Hence, decreasing sample size requires a downsizing of the cantilever as well (Reiche et al., 2015a). 
The cantilever's motion is usually detected by laser interferometry or laser deflection (Buchter et al., 2013; Lavenant et al., 2014), which becomes increasingly difficult if the cantilever dimensions are reduced. Therefore, we have two contradicting requirements: on the one hand the sensitivity needs to be increased in order to measure weaker signals and on the other hand maintaining the ease of detection. We are addressing these two aims simultaneously with a so-called coresonant sensor concept. This concept is based on two cantilever beams, one being of micrometre size and the other one having nanometre size (at least for two out of three dimensions), which are coupled in succession to each other. The coresonance is introduced by matching the individual eigenfrequencies of the otherwise very different cantilevers. Please note that the sensor concept itself is of a general nature; however, here we are applying it to two cantilever beams and match the eigenfrequencies of the first flexural vibration modes.

For our experiments we used a commercially available atomic force microscopy (AFM) microcantilever and a carbon nanotube (CNT) as nanocantilever. Frequency matching between the two subsystems has been achieved by adding some mass to the free end of the nanocantilever. The frequency matching introduces a very strong interplay between the two subsystems, resulting in two main effects. First, a significant amplitude amplification between micro- and nanocantilever is observed, leading to very high oscillation amplitudes of the nanocantilever ( $\mathrm{Li}$ et al., 2003; VidalÁlvarez et al., 2015). Second, the strong interplay allows for a determination of changes in the oscillatory state of the coupled system which are due to interactions between nanocantilever and environment, in the amplitude response curve of the microcantilever. This allows for an easy detection of the oscillatory state of a nanocantilever, which has been described as one of the challenges when employing nanocantilevers as sensors (Philippi et al., 2011). Please note that there are detection methods available for direct measurement of a nanocantilever's oscillation as for example shown by Gil-Santos et al. (2010). These methods usually require very sophisticated experimental set-ups, whereas our co-resonantly coupled sensors are compatible with standard set-ups that are used for excitation and detection of microcantilever oscillations, e.g. atomic force microscopes.

The behaviour of the co-resonant sensor concept has been studied thoroughly in terms of analysing the mechanical model and the system's differential equations (Reiche et al., 2015a). Furthermore, we have already employed the sensor for cantilever magnetometry measurements, which proved a significant increase in sensitivity compared to magnetometry experiments with similar magnetic samples (Körner et al., 2015, 2016). However, the purely mechanical description of the system is only one possibility. Another way of studying the complex behaviour of the coupled system is given by translating it in an electrical circuit. This facilitates the

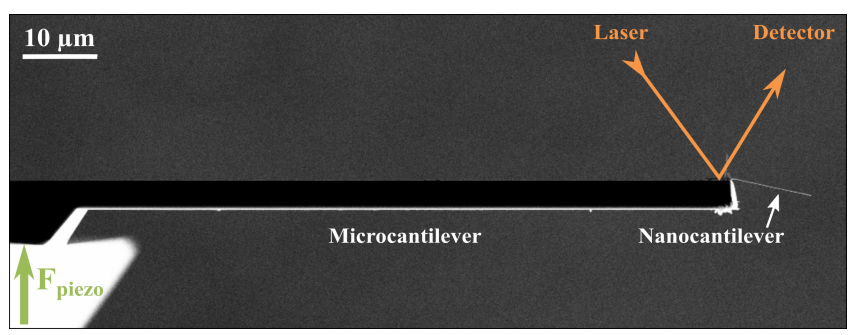

Figure 1. Example of a coupled sensor consisting of a micro- and a nanocantilever. The sensor is driven with a piezoelectric actuator, and the oscillation is detected with laser deflection.

sensor characterization as well as the evaluation of measurements by allowing the use of network analysis tools.

In the following, we will derive an electric circuit model for the sensor and discuss its behaviour for various simplifications. Analytical expressions will be given, which are evaluated with the software Mathematica if necessary. In terms of circuit analysis, the software LTspice is used. Furthermore, we will compare the obtained results with experimental data for a co-resonantly coupled sensor, which is discussed in detail in Körner et al. (2016). The sensor's properties are therefore given in Table 2. In addition, a scanning electron microscope picture of the sensor with the principle measurement set-up is shown in Fig. 1. Please note that this is only one representation of the co-resonant sensor concept and that other geometries are possible as well, for example with the nanocantilever being positioned perpendicular to the microcantilever (Reiche et al., 2015b).

\section{Electric circuit model of the co-resonant sensor}

The sensor employs two cantilevers which are coupled in succession as depicted in Fig. 1. It is well known that a oneside clamped beam exhibits an infinite number of eigenfrequencies (Rossing and Fletcher, 2004). However, for using it as a sensor element, it will only be driven at or close to one of its eigenfrequencies, allowing for the description as a harmonic oscillator (Rast et al., 2000). This assumption holds for both of the cantilevers of the coupled system. In order to take interactions between the sensor and its environment into account, an additional spring $k_{3}$ and damping element $d_{3}$ are included, so that the mechanical model depicted in Fig. 2 can be derived. Furthermore, the sensor is excited to oscillate through a piezoelectric actuator. It is not necessary to model this actuator by an electric circuit itself in order to understand the coupled system, but instead we will consider it as a periodic displacement on the clamping point of the microcantilever. This can be translated into a periodic force acting on the mass $m_{1}$ as it is depicted in Fig. 2.

Given the mechanical representation of the sensor, we can derive the electric circuit model by employing the analogies in Table 1 (Lenk et al., 2001). Please note that these analogies 


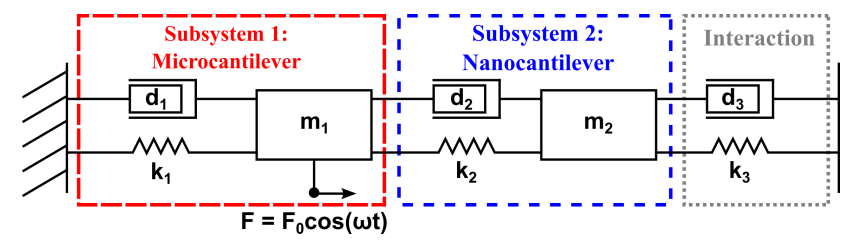

Figure 2. Mechanical model for the sensor depicted in Fig. 1.

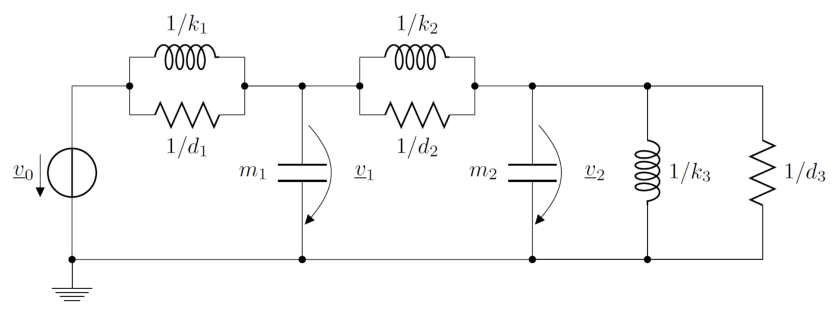

Figure 3. Electric circuit model for a co-resonant cantilever sensor whose mechanical representation is shown in Fig. 2.

are not the only ones possible but are commonly used to describe the behaviour of dynamically excited beams (Hähnle, 1931).

With these analogies, the structure of the mechanical representation is preserved with only the masses being treated specifically as one of their connection points always has to be the frame, hence ground in the electric case (Klotter, 1950). The resulting electric representation is depicted in Fig. 3. Please note that the piezo actuator's effect is that of a periodic displacement of the microcantilever: $A(t)=A_{0} \sin (\omega t)$, which is recalculated into a periodic velocity by $v_{0}(t)=$ $\partial A(t) / \partial t=A_{0} \omega \cos (\omega t)$. Hence, the voltage source is on the left-hand side of the circuit in Fig. 3.

The sensor concept itself is of a general nature, but in order to show some quantitative evaluation of the following calculations, the mechanical properties of one fabricated coresonant sensor are given in Table 2, as well as the corresponding values for the electric elements. Since we do not want to discuss the absolute values for the oscillation amplitude of the sensor, we did not use a conversion factor for calculating the electric elements as it is suggested by others (Ballas et al., 2009) but instead simply used the analogies in Table 1. The values for the mechanical elements have been determined from experiments (Körner et al., 2015), and the mass $m_{i}$ of each cantilever beam equals the effective mass given by the beam's spring constant $k_{i}$ and its eigenfrequency $f_{i}$ after the frequency matching process:

$m_{i}=k_{i} /\left(2 \pi f_{i}\right)^{2}$.

The damping constants $d_{i}$ are derived from the mechanical quality factor $Q_{i}$ by

$d_{i}=\sqrt{m_{i} k_{i}} / Q_{i}$.

Since the eigenfrequencies $f_{1,2}$ and quality factors $Q_{1,2}$ of the two cantilevers are needed for calculating the effective
Table 1. Analogies used for the conversion of mechanical systems into an electric circuit.

\begin{tabular}{ll}
\hline Mechanical & Electrical \\
\hline Force $F$ & Current $I$ \\
Velocity $v$ & Voltage $U$ \\
Mass $m$ & Capacitor $C \equiv m$ \\
Spring $k$ & Inductor $L \equiv 1 / k$ \\
Damper $d$ & Resistor $R \equiv 1 / d$ \\
\hline
\end{tabular}

Table 2. Values for the mechanical and electrical elements, the latter being calculated according to Table 1 .

\begin{tabular}{lll}
\hline Element & Mechanical & Electrical \\
\hline Eigenfrequency $f_{1}$ & $(723080 \pm 1) \mathrm{Hz}$ & $723080 \mathrm{~Hz}$ \\
Spring $k_{1}$ & $(133.8 \pm 8) \mathrm{Nm}^{-1}$ & $0.0075 \mathrm{H}$ \\
$Q$-factor $Q_{1}$ & $(3390 \pm 100)$ & 3390 \\
Mass $m_{1}$ & $(6.5 \pm 0.4) \times 10^{-12} \mathrm{~kg}$ & $6.5 \times 10^{-12} \mathrm{~F}$ \\
Damper $d_{1}$ & $(8.7 \pm 0.8) \times 10^{-9} \mathrm{~kg} \mathrm{~s}^{-1}$ & $1.15 \times 10^{8} \Omega$ \\
\hline Eigenfrequency $f_{2}$ & $(725610 \pm 1) \mathrm{Hz}$ & $725610 \mathrm{~Hz}$ \\
Spring $k_{2}$ & $(0.009 \pm 0.005) \mathrm{Nm}^{-1}$ & $116.74 \mathrm{H}$ \\
$Q$-factor $Q_{2}$ & $(450 \pm 50)$ & 450 \\
Mass $m_{2}$ & $(4.1 \pm 2.7) \times 10^{-16} \mathrm{~kg}$ & $4.12 \times 10^{-16} \mathrm{~F}$ \\
Damper $d_{2}$ & $(4.2 \pm 3) \times 10^{-12} \mathrm{~kg} \mathrm{~s}^{-1}$ & $2.4 \times 10^{11} \Omega$ \\
\hline
\end{tabular}

masses $m_{1,2}$ and the damping $d_{1,2}$ in the mechanical model, they are also given in Table 2, although they are not needed in the circuit model. The experimental determination of the quality factor and damping has been done for each cantilever before the frequency matching. Please note that the eigenfrequencies $f_{1,2}$ are given for the matched state.

At this point it is useful to review the terms eigenfrequency and resonance frequency. The eigenfrequency is the frequency where the maximum amplitude occurs for an undamped driven harmonic oscillator. For the case of a damped driven harmonic oscillator the frequency where the maximum amplitude occurs is termed resonance frequency (Tipler and Mosca, 2015, p. 440). Please note that this is only one possible distinction and that for systems with low damping these terms are commonly used interchangeably. Throughout this paper we will be using the term resonance frequency for all discussions related to the coupled system. The term eigenfrequency is only used when talking about the single cantilevers in order to distinguish their properties from the coupled system. In addition, frequency values are given for the experimental case and all numerical information, whereas all mathematical expressions include the angular frequency $\omega$, related to the frequency $f$ by $\omega=2 \pi f$. 


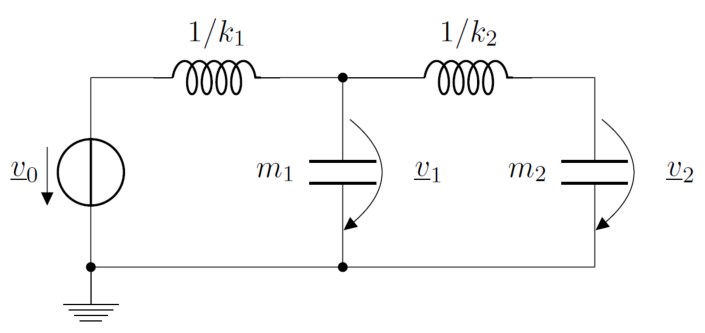

Figure 4. Simplified circuit model from Fig. 3 without damping and external interaction, i.e. $d_{i}=0, k_{3}=0$.

\section{Circuit model without damping and external interaction}

The simplest case we will consider is the circuit from Fig. 3 where we neglect any damping, i.e. $d_{1}=d_{2}=d_{3}=0$, and the external interaction $k_{3}=0$, therefore reducing it to the rather simple structure shown in Fig. 4.

The quantities of interest are the velocities $\underline{v}_{1}$ and $\underline{v}_{2}$, i.e. the voltages over the capacitors, as the ratios $v_{1} / v_{0}$ and $v_{2} / v_{0}$ correspond directly to the amplitudes of the oscillating cantilever beams in the mechanical system. ${ }^{1}$ The expressions for $\underline{v}_{1}$ and $\underline{v}_{2}$ can be found analytically by applying the rule for voltage division. To shorten the expressions the symbol ॥ is used to represent a parallel circuit:

$$
\begin{aligned}
& \frac{\underline{v}_{1}}{\underline{v}_{0}}=\frac{\frac{1}{j \omega m_{1}} \|\left(\frac{1}{j \omega m_{2}}+j \omega \frac{1}{k_{2}}\right)}{\frac{1}{j \omega m_{1}} \|\left(\frac{1}{j \omega m_{2}}+j \omega \frac{1}{k_{2}}\right)+j \omega \frac{1}{k_{1}}} \\
& \underline{\underline{v}}_{2}=\frac{1}{1-\omega^{2}\left(\frac{m_{2}}{k_{2}}\right)} \underline{\underline{v}}_{0} .
\end{aligned}
$$

Rearranging and using the relation $\omega_{i}^{2}=k_{i} / m_{i}, i \in[1,2]$ yields

$$
\begin{aligned}
& A_{1}(\omega)=\left|\frac{\underline{v}_{1}}{\underline{v}_{0}}\right|=\frac{v_{1}}{v_{0}}=\frac{1}{1-\left(\frac{\omega}{\omega_{1}}\right)^{2}-\frac{m_{2}}{m_{1}} \cdot \frac{\left(\omega / \omega_{1}\right)^{2}}{1-\left(\omega / \omega_{2}\right)^{2}}} \\
& A_{2}(\omega)=\left|\frac{\underline{v}_{2}}{\underline{v}_{0}}\right|=\frac{v_{2}}{v_{0}}=\frac{1}{1-\left(\frac{\omega}{\omega_{2}}\right)^{2}} \cdot \frac{v_{1}}{v_{0}} .
\end{aligned}
$$

These expressions are evaluated for the numerical values given in Table 2, and the resulting curves are depicted in Fig. 5. The coupled system exhibits two distinct resonances $\omega_{a}$ and $\omega_{b}$ due to the closely matched eigenfrequencies $\omega_{1}$ and $\omega_{2}$ of the two cantilevers. However, since no damping is considered, the amplitudes reach infinite values at the resonance frequencies $\omega_{a}$ and $\omega_{b}$, which is not the case for a real system. Nevertheless, it is possible to calculate these poles of

\footnotetext{
${ }^{1}$ Underscores will be used throughout the text to indicate variables with complex values, and variables without underscores denote the magnitude.
}
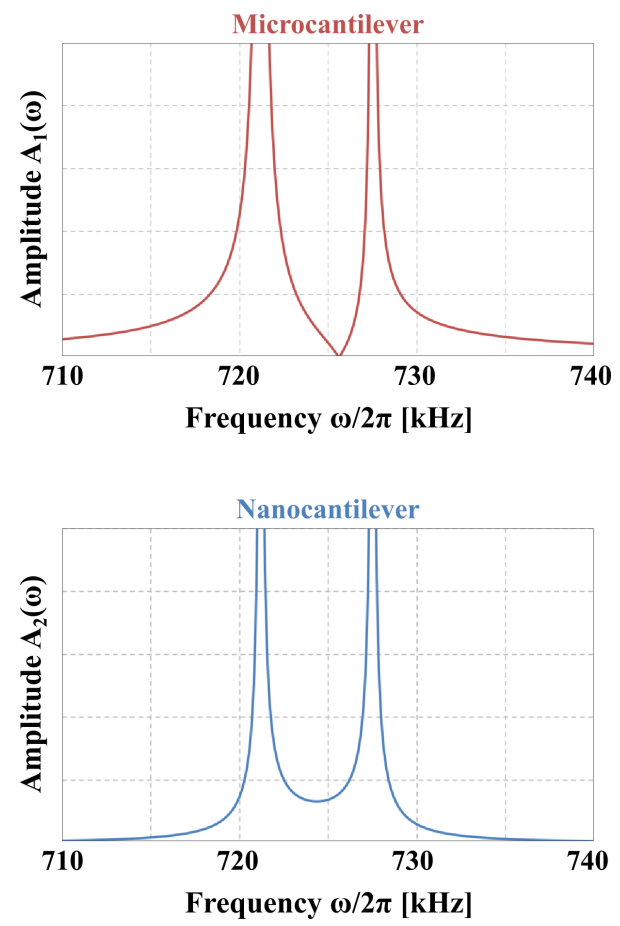

Figure 5. Amplitude curves of both cantilevers of the circuit from Fig. 4 without damping and external interaction, according to Eqs. (5) and (6).

the amplitude curves by evaluating the denominator for the two expressions in Eqs. (5) and (6). In both cases we arrive at the same formula, giving the two resonance frequencies of the coupled system. We will denote them with $\omega_{a}$ and $\omega_{b}$ (or $f_{a}$ and $f_{b}$ ) in the following to avoid confusion with the eigenfrequencies $\omega_{1}$ and $\omega_{2}$ (or $f_{1}$ and $f_{2}$ ) of the individual subsystems. The poles are given by

$$
\begin{aligned}
\omega_{a, b} & = \pm \sqrt{\frac{B_{1} \pm \sqrt{B_{1}^{2}-4 B_{2}}}{2 B_{2}}} \\
B_{1} & =\frac{m_{2}}{m_{1} \omega_{1}^{2}}+\frac{1}{\omega_{2}^{2}}+\frac{1}{\omega_{1}^{2}} \\
B_{2} & =\frac{1}{\omega_{1}^{2} \omega_{2}^{2}} .
\end{aligned}
$$

Please note that the complete solution for $\omega_{a, b}$ also contains negative frequencies, but they are not considered since they do not correspond to actual frequency values. The comparison of the coupled system's resonance frequencies $\omega_{a, b}$ to the eigenfrequencies $\omega_{1,2}$ of the subsystems shows that the coupled frequencies are further apart than the eigenfrequencies. Furthermore, comparing the calculated values to measurements (see Table 3), we observe that the values agree within the margins of uncertainty. 
Table 3. Comparison of calculated and measured resonance frequencies $f_{a}$ and $f_{b}$ of the coupled system represented by the circuit in Fig. 4.

\begin{tabular}{rrr}
\hline & Calculated & Measured \\
\hline$f_{a}$ & $(721.2 \pm 0.6) \mathrm{kHz}$ & $(720.68 \pm 0.01) \mathrm{kHz}$ \\
$f_{b}$ & $(727.5 \pm 0.7) \mathrm{kHz}$ & $(728.05 \pm 0.07) \mathrm{kHz}$ \\
\hline
\end{tabular}
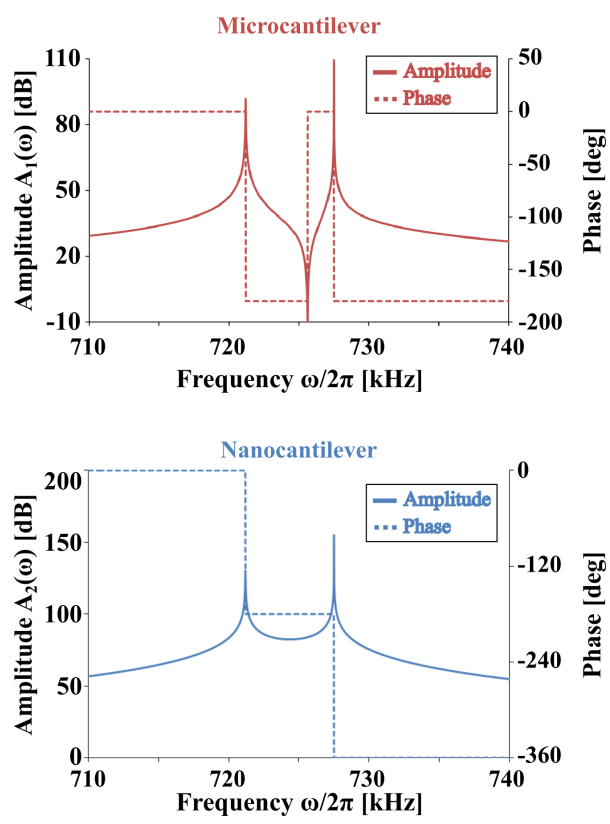

Figure 6. Bode plots for both subsystems of Fig. 4 without damping and external interaction obtained with the circuit analysis software LTspice.

The electric circuit given in Fig. 4 has also been evaluated with LTspice in order to obtain a Bode plot (Dorf and Svoboda, 2010), which is shown in Fig. 6 for $A_{1}$ and $A_{2}$. In this form of representation, $A_{1}(\omega)$, i.e. the amplitude response curve for the microcantilever, shows a so-called antiresonance at the eigenfrequency $\omega_{2}$ of the nanocantilever. At an antiresonance point, the oscillation of the microcantilever would go to zero due to the presence of the nanocantilever (Joe et al., 2006). This behaviour is well known and used in vibration damping (Axisa, 2004). For a more detailed discussion on antiresonance please refer to Belbasi et al. (2014).

With this simplified circuit model it is possible to obtain the resonance frequencies as well as find typical features of the coupled system like frequency shifting compared to the eigenfrequencies of the subsystems and antiresonance behaviour.
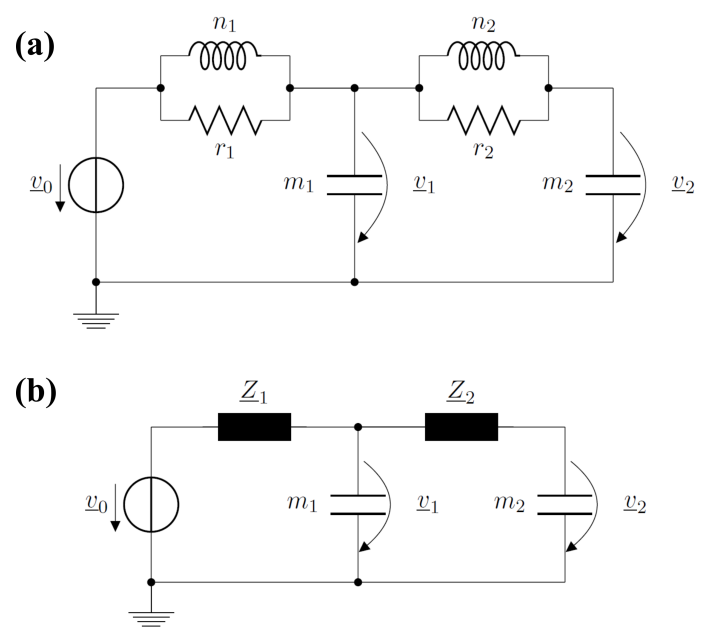

Figure 7. Circuit model with damping and without external interaction, (a) complete, (b) with parallel branches combined into complex impedances $\underline{Z}_{1,2}$.

\section{Circuit model with damping and without external interaction}

\subsection{Amplitude and phase relations}

The model so far does not represent the finite amplitudes of the resonance peaks; therefore, the expected amplitude amplification between the nano- and the microcantilever cannot be studied. Hence, we will include the damping in this section and derive an analytical expression for this case. We again consider a coupled system without external interaction, i.e. $d_{3}=0$ and $k_{3}=0$, but include $d_{1}$ and $d_{2}$. Furthermore, we will be using the relations $n_{i}=1 / k_{i}$ and $r_{i}=1 / d_{i}, i \in$ $[1,2]$ to facilitate the resulting expressions, leading to the circuit depicted in Fig. 7a. Adding the two resistors results in two new parallel branches of the circuit. In order to facilitate the analytical derivation, we combine each of the parallel branches in a complex impedance $\underline{Z}_{1}$ and $\underline{Z}_{2}$ as shown in Fig. 7b. The resulting expressions become

$\underline{Z}_{i}=\frac{j \omega n_{i} r_{i}}{r_{i}+j \omega n_{i}}$ 
With that we can again apply the rule for voltage division to derive expressions for $\underline{v}_{1}$ and $\underline{v}_{2}$ :

$\frac{\underline{v}_{1}}{\underline{v}_{0}}=\frac{R_{N}^{1}+j X_{N}^{1}}{R_{D}+j X_{D}}$
$\frac{\underline{v}_{2}}{\underline{v}_{0}}=\frac{R_{N}^{2}+j X_{N}^{2}}{R_{D}+j X_{D}}$

with the following substitutions

$R_{N}^{1}=r_{1} r_{2} \Omega_{2}-\omega^{2} n_{1} n_{2}$

$R_{N}^{2}=r_{1} r_{2}-\omega^{2} n_{1} n_{2}$

$R_{D}=r_{1} r_{2}\left[\Omega_{2}-\left(\frac{\omega}{\omega_{1}}\right)^{2}\left(\frac{m_{2}}{m_{1}}+\Omega_{2}\right)\right]-\omega^{2} n_{1} n_{2}$

$X_{N}^{1}=\omega\left[r_{1} n_{2}+r_{2} n_{1} \Omega_{2}\right]$

$X_{N}^{2}=\omega\left[n_{1} r_{2}+n_{2} r_{1}\right]$

$X_{D}=\omega\left[r_{1} n_{2} \Omega_{1}+r_{2} n_{1} \Omega_{2}-r_{1} n_{1}\left(\frac{\omega}{\omega_{2}}\right)^{2}\right]$

$\Omega_{1}=1-\left(\frac{\omega}{\omega_{1}}\right)^{2}$

$\Omega_{2}=1-\left(\frac{\omega}{\omega_{2}}\right)^{2}$.

This time the expressions are complex, but by separating the real and imaginary parts of numerator and denominator and applying the rules for complex numbers (Bronstein et al., $2005)$ it is possible to obtain the amplitude $A_{i}(\omega)=\left|\underline{v}_{i} / \underline{v}_{0}\right|$ and phase relations for both expressions.

$A_{1}(\omega)=\left|\frac{\underline{v}_{1}}{\underline{v}_{0}}\right|=$

$\frac{\sqrt{\left(R_{N}^{1} R_{D}+X_{N}^{1} X_{D}\right)^{2}+\left(X_{N}^{1} R_{D}-R_{N}^{1} X_{D}\right)^{2}}}{R_{D}^{2}+X_{D}^{2}}$

$A_{2}(\omega)=\left|\frac{\underline{v}_{2}}{\underline{v}_{0}}\right|=$

$\frac{\sqrt{\left(R_{N}^{2} R_{D}+X_{N}^{2} X_{D}\right)^{2}+\left(X_{N}^{2} R_{D}-R_{N}^{2} X_{D}\right)^{2}}}{R_{D}^{2}+X_{D}^{2}}$

$\tan \left(\Phi_{1}(\omega)\right)=\left(\frac{X_{N}^{1} R_{D}-R_{N}^{1} X_{D}}{R_{N}^{1} R_{D}+X_{N}^{1} X_{D}}\right) \Phi_{1} \in(-\pi, 0)$

$\tan \left(\Phi_{2}(\omega)\right)=\left(\frac{X_{N}^{2} R_{D}-R_{N}^{2} X_{D}}{R_{N}^{2} R_{D}+X_{N}^{2} X_{D}}\right) \Phi_{2} \in(-2 \pi, 0)$

In this work we focus on the amplitude expressions since the amplitude response is used as the main measurement signal in our experiments. In case the phase of the oscillation becomes important, for example if a phase-locked loop is used to track the change of the resonance frequencies of the
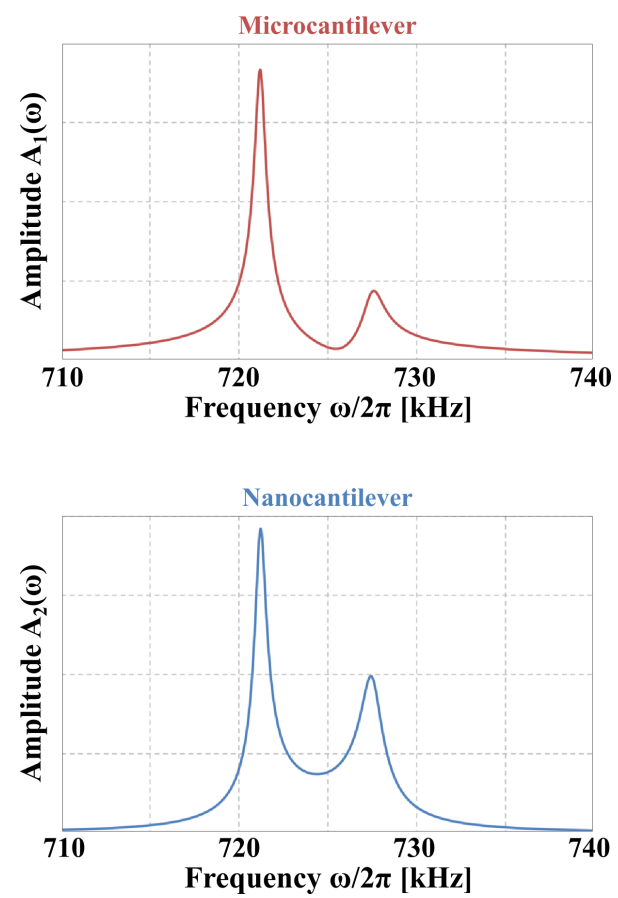

Figure 8. Amplitude curves obtained with Eqs. (21) and (22) for both subsystems of the circuit model with damping and without external interaction from Fig. 7.

Table 4. Calculated resonance frequencies $f_{a, b}$ of the coupled system for both subsystems obtained with the circuit model of Fig. 7 that includes damping. Furthermore the frequency $f_{\min }$, which corresponds to the amplitude minimum between the two resonance peaks is given.

\begin{tabular}{lr|r}
\hline & \multicolumn{2}{c}{ Calculation based on } \\
\cline { 2 - 3 } & $A_{1}(\omega)$ & $A_{2}(\omega)$ \\
\hline$f_{a}$ & $(721.2 \pm 0.4) \mathrm{kHz}$ & $(721.2 \pm 0.4) \mathrm{kHz}$ \\
$f_{b}$ & $(727.6 \pm 0.4) \mathrm{kHz}$ & $(727.4 \pm 0.4) \mathrm{kHz}$ \\
$f_{\text {min }}$ & $(725.5 \pm 0.4) \mathrm{kHz}$ & $(724.4 \pm 0.4) \mathrm{kHz}$ \\
\hline
\end{tabular}

coupled system, the corresponding phase relations are given for completeness. With these phase relations, one is able to conduct a similar discussion as follows for the amplitude expressions. Plotting Eqs. (21) and (22) with the parameters of the real sensor gives the amplitude curves depicted in Fig. 8. With the resonant amplitudes reaching finite values it is possible to study the behaviour of the coupled system in more detail.

First, the two resonance frequencies $\omega_{a}$ and $\omega_{b}$ are obtained by setting the derivatives of Eqs. (21) and (22) to zero. The software Mathematica has been used for this and all following calculations in this work. The results for the resonance frequencies for both peaks and evaluation of $A_{1}(\omega)$ and $A_{2}(\omega)$ are given in Table 4 . They barely differ from the ones obtained with the simplified model without damping. 

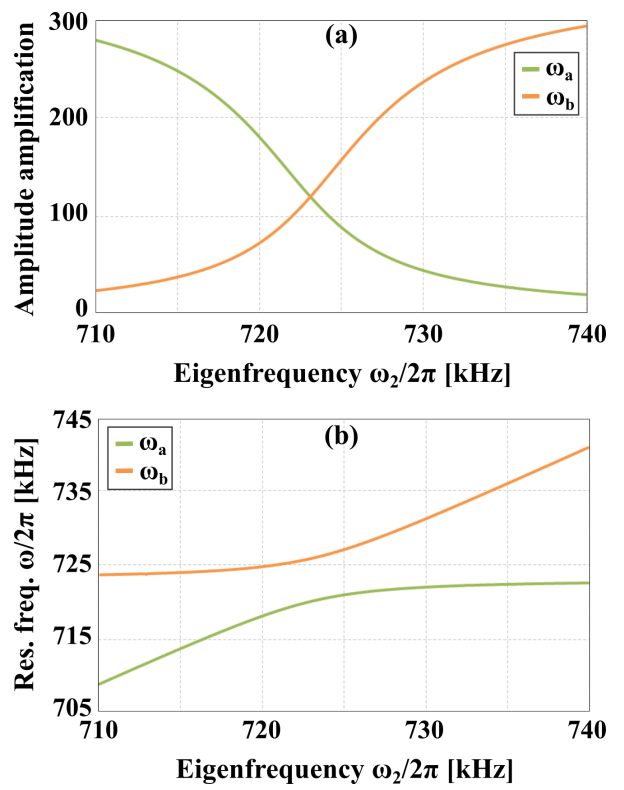

Figure 9. (a) Amplitude amplification factor for both resonance peaks and (b) resonance frequencies of the coupled system with varying eigenfrequency $\omega_{2}=2 \pi f_{2}$ of the nanocantilever.

Furthermore, it is also possible to find the frequency $f_{\min }$ value for the minimum amplitude in between the two resonance peaks. In case of $A_{1}(\omega)$, i.e. the microcantilever, this frequency again corresponds to the antiresonance state of its oscillation. However, due to the damping, the amplitude does not go to zero anymore (Belbasi et al., 2014) but the effect is merely an asymmetric shape of the resonance curve.

\subsection{Amplitude amplification}

In addition, it is possible to study the amplitude ratio between the amplitudes of nano- and microcantilever, which we will term amplitude amplification in the following. The amplitude of the nanocantilever is increased compared to that of the microcantilever due to the co-resonant coupling; the amplitude amplification factor $\Lambda=A_{2} / A_{1}$ can be obtained, which is a function of the distance of the subsystem's eigenfrequencies. In general, one could simply calculate $A_{2}(\omega) / A_{1}(\omega)$ for all $\omega$. But in that case, the maximum amplitude amplification would occur close to the antiresonance frequency of subsystem 1 where its amplitude $A_{1}$ is minimal. From the experimental point of view, only the amplitude amplification at the resonance peaks of the microcantilever, i.e. $A_{1}\left(\omega_{a}\right)$ and $A_{1}\left(\omega_{b}\right)$, is of interest since this would be measured for a sensor. Hence, we will discuss the amplification accordingly.

A general approach to find the amplitude amplification factor is to determine the resonance frequencies $\omega_{a}$ and $\omega_{b}$ of the coupled system, which then leads to two amplification factors, namely $\Lambda_{1}^{a}=A_{2}\left(\omega_{a}\right) / A_{1}\left(\omega_{a}\right)$ and $\Lambda_{1}^{b}=$ $A_{2}\left(\omega_{b}\right) / A_{1}\left(\omega_{b}\right)$ of the two resonance peaks of subsystem 1 . These calculations have been carried out for $\omega_{1}=$ constant
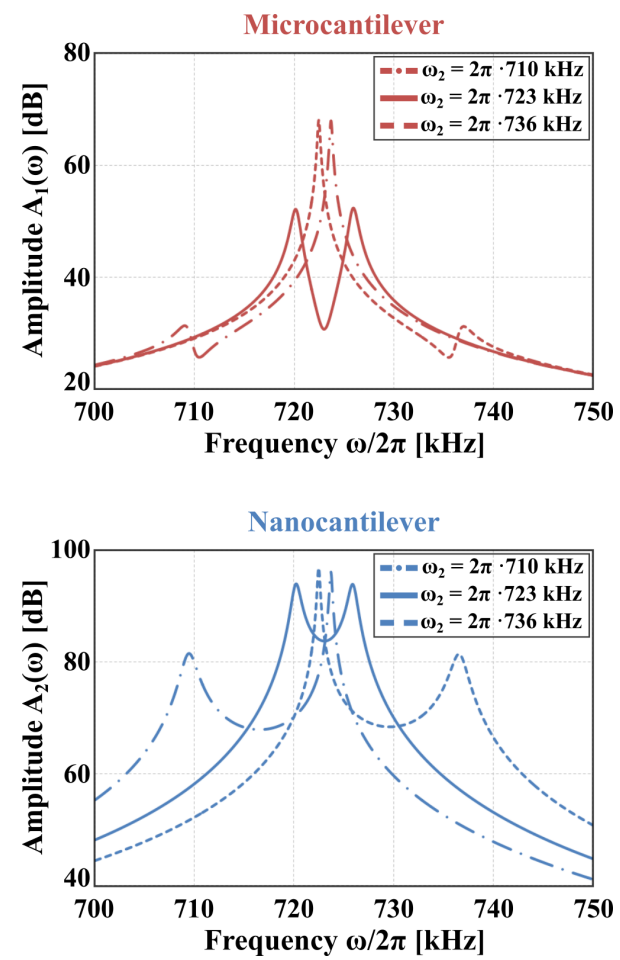

Figure 10. Bode plot of amplitude curves for both cantilevers of the coupled system from Fig. 7 for varying $\omega_{2}=2 \pi f_{2}$.

and varying $\omega_{2}$ for the values of the sensor given in Table 2 . The eigenfrequency $\omega_{2}$ of the nanocantilever has been changed from $2 \pi \times 710$ to $2 \pi \times 740 \mathrm{kHz}$. The resulting amplitude amplification curve for both resonance peaks of subsystem 1 is depicted in Fig. 9a as well as the dependence $\omega_{a}\left(\omega_{2}\right)$ and $\omega_{b}\left(\omega_{2}\right)$ in Fig. 9b. Please note that $\omega_{a}$ always denotes the lower resonance frequency, i.e. the left-hand side peak and $\omega_{b}$ the resonance peak at the higher frequency. From Fig. 9b it is obvious that the two resonance frequencies are approaching each other and get closest when $\omega_{2} \approx \omega_{1}$ but then diverge again without ever crossing. This behaviour is called "avoided crossing" and is well known from discussions of coupled harmonic oscillator models describing quantum systems (Novotny, 2010; Rubbmark et al., 1981).

When looking at Fig. 9a, one has to keep in mind the practical relevance of the amplitudes of the two resonance peaks in order to understand the amplification induced by the coupled system. To clarify this point, Fig. 10 depicts amplitude curves $A_{1}(\omega)$ and $A_{2}(\omega)$ for several values of $\omega_{2}$. From these we see that for the cases $\omega_{2}<\omega_{1}$ and $\omega_{2}>\omega_{1}$ (dashed lines in Fig. 10) the resonance peak close to the eigenfrequency $\omega_{2}$ of the nanocantilever in $A_{1}$ is very small, whereas in $A_{2}$ it is significant since $A_{2}$ describes the amplitude of the nanocantilever. Hence, the amplitude amplification would be very high for this comparatively small peak, corresponding to the upper left branch in Fig. 9a. In terms of measuring, this is not sensible since one would usually track the peak with the 


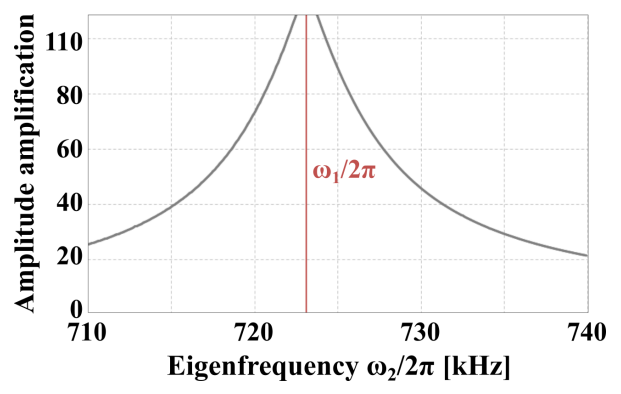

Figure 11. Amplification factor for the higher resonance peak of the coupled system for varying eigenfrequency $\omega_{2}=2 \pi f_{2}$ of the nanocantilever. The eigenfrequency $\omega_{1}=2 \pi f_{1}$ of the microcantilever is kept constant and is indicated by the vertical line.

higher amplitude and, due to noise of the measurement setup, the smaller peak might not even be visible. Therefore, from an experimental point of view, only the amplitude amplification factor represented by the lower left branch of the curve in the diagram in Fig. 9a is considered. The same reasoning can be applied for the two branches on the right-hand side of Fig. 9a. Conclusively, when tracking the resonance peak with the higher amplitude, one can obtain the amplitude amplification factor depending on $\omega_{2}$ depicted in Fig. 11. The curve has been cut off for an amplification higher than 110 since - for very close eigenfrequencies $\omega_{1}$ and $\omega_{2}$ of the two subsystems - the two resonance peaks reach a similar amplitude.

\subsection{Analytical determination of the eigenfrequency of the nanocantilever}

In order to describe the coupled systems' behaviour with the circuit model, the properties of the subsystems have to be known. They can be determined from their geometric and material properties as well as from measurements. However, it is particularly difficult to determine the eigenfrequency $\omega_{2}$ of the nanocantilever, and during sensor production it is only possible to make a rough estimate of it. Nevertheless, in order to use the sensor for quantitative measurements, it is necessary to know the eigenfrequencies $\omega_{1}$ and $\omega_{2}$ of both subsystems as accurate as possible. By using the above formula describing the amplitude curve of the coupled system measured at the microcantilever Eq. (21), the eigenfrequency $\omega_{2}=2 \pi f_{2}$ of the nanocantilever can be estimated rather accurately. Besides the properties of the two cantilevers (spring constant, damping, effective mass), the eigenfrequency $\omega_{1}$ of the microcantilever - as well as one of the resonance frequencies of the coupled system $\omega_{a}$ or $\omega_{b}-$ is required. These frequencies can easily be obtained from measurements.

Applying these values in Eq. (21) for the amplitude response curve of the microcantilever, the amplitude $A_{1}$ can be described dependent on the eigenfrequency $\omega_{2}$ of the nanocantilever instead of the frequency $\omega$, which is fixed to $\omega_{a}$ or $\omega_{b}$. The frequency where the maximum of $A_{1}\left(\omega_{2}\right)$ occurs corresponds to the eigenfrequency $\omega_{2}$ of the nanocantilever. The maximum may be obtained numerically or analytically, in the latter case by setting the derivative of the amplitude curve $A_{1}\left(\omega_{2}\right)$ to zero and calculating the frequencies for which the second derivative of the amplitude curve is negative. The numerical values of our sensor demonstrate the applicability of this approach. The eigenfrequency of the nanocantilever has been determined experimentally to be $f_{2} \approx(726250 \pm 100) \mathrm{Hz}$ right after sensor fabrication. Handling the sensor, which included exposure to air and the transfer into another machine for the magnetic measurements, is expected to alter the eigenfrequency $\omega_{2}$, which is proved by a change of the coupled system's resonance frequencies $\omega_{a}$ and $\omega_{b}$ as well. Therefore, the above expressions have been used to determine the nanocantilever's eigenfrequency and $f_{2} \approx(725610 \pm 500) \mathrm{Hz}$ is obtained, which is in good agreement with the expected decrease of the eigenfrequency due to exposure to air (Botman et al., 2008).

\section{Circuit model with damping and external interaction}

The most general description of the coupled sensor is the one depicted in Fig. 3 with all elements including the external interaction modelled by the spring $k_{3}$ and the damping element $d_{3}$. It is possible to derive analytical expressions for $\underline{v}_{1} / \underline{v}_{0}$ and $\underline{v}_{2} / \underline{v}_{0}$ by the following steps.

First, parallel branches are combined into complex impedances to simplify the circuit. As depicted in Fig. 3, the elements $m_{2}, k_{3}$ and $d_{3}$ lie in parallel with the same voltage, i.e. velocity, $\underline{v}_{2}$ over them. Hence, these elements are combined into the impedance $\underline{Z}_{3}$, advantageously written in the Euler form for complex numbers, i.e. $\underline{Z}_{3}=Z_{3} \cdot e^{j \varphi_{3}}$. Furthermore, using $n_{3}=1 / k_{3}$ and $r_{3}=1 / d_{3}$ gives

$$
\begin{aligned}
& Z_{3}=\frac{\sqrt{\left(\omega^{2} n_{3}^{2} r_{3}\right)^{2}+\left(\omega n_{3} r_{3}^{2}\left(1-\omega^{2} m_{2} n_{3}\right)\right)^{2}}}{\left(r_{3}-\omega^{2} m_{2} n_{3} r_{3}\right)^{2}+\left(\omega n_{3}\right)^{2}} \\
& \varphi_{3}=\arctan \left(\frac{r_{3}\left(1-\omega^{2} m_{2} n_{3}\right)}{\omega n_{3}}\right) .
\end{aligned}
$$

The same is done for the parallel branches of $r_{1}, n_{1}$ and $r_{2}, n_{2}$, now represented by the complex impedances $\underline{Z}_{i}=Z_{i}$. $e^{j \varphi_{i}}, i \in[1,2]$ :

$$
\begin{aligned}
Z_{i} & =\frac{\sqrt{\left(\omega^{2} n_{i}^{2} r_{i}\right)^{2}+\left(\omega n_{i} r_{i}^{2}\right)^{2}}}{r_{i}^{2}+\left(\omega n_{i}\right)^{2}} \\
\varphi_{i} & =\arctan \left(\frac{r_{i}}{\omega n_{i}}\right) .
\end{aligned}
$$

With these transformations we get the circuit depicted in Fig. 12. In a next step, the sum of the impedances $\underline{Z}_{23}=$ 


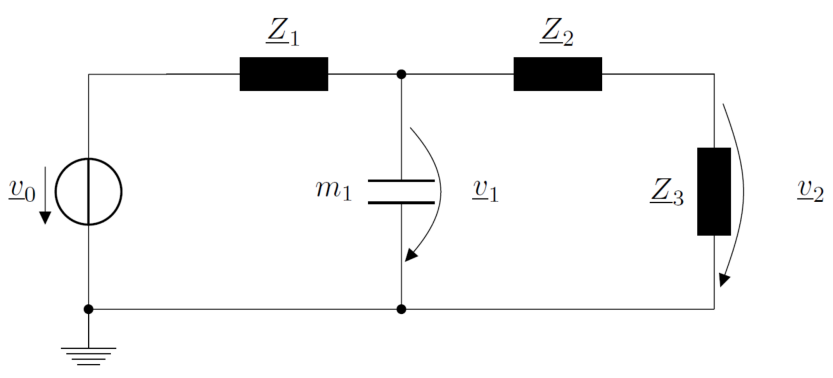

Figure 12. Simplified circuit model for Fig. 3 to derive analytical expressions for $\underline{v}_{1}$ and $\underline{v}_{2}$ in case of a given external interaction $k_{3}, d_{3}$ at the nanocantilever.

$\underline{Z}_{2}+\underline{Z}_{3}$ is calculated:

$Z_{23}=$

$\sqrt{\omega^{4}\left(\frac{n_{2}^{2} r_{2}}{N_{2}}+\frac{n_{3}^{2} r_{3}}{N_{3}}\right)^{2}+\omega^{2}\left(\frac{n_{2} r_{2}^{2}}{N_{2}}+\frac{n_{3} r_{3}^{2}\left(1-\omega m_{2} n_{3}\right)}{N_{3}}\right)^{2}}$,

$\varphi_{Z_{23}}=\arctan \left(\frac{n_{2} r_{2}^{2} N_{3}+n_{3} r_{3}^{2} N_{2}\left(1-\omega^{2} m_{2} n_{3}\right)}{\omega\left(n_{2}^{2} r_{2} N_{3}+n_{3}^{2} r_{3} N_{2}\right)}\right)$

with

$N_{2}=r_{2}^{2}+\left(\omega n_{2}\right)^{2} \quad$ and

$N_{3}=\left(r_{3}-\omega^{2} m_{2} n_{3} r_{3}\right)^{2}+\left(\omega n_{3}\right)^{2}$.

In order to express all elements of the circuit in Euler form so that the following calculations will be facilitated, the capacitor $m_{1}$ will also be described by an impedance $\underline{Z}_{m}=Z_{m} \cdot e^{j \varphi_{m}}$ :

$Z_{m}=\frac{1}{\omega m_{1}} ; \quad \varphi_{m}=\frac{\pi}{2}$.

With the above definitions and combinations it is now possible to give expressions for $\underline{v}_{1} / \underline{v}_{0}$ and $\underline{v}_{2} / \underline{v}_{0}$.

$\underline{A}_{1}=\frac{\underline{v}_{1}}{\underline{v}_{0}}=\frac{\underline{Z}_{m} \| \underline{Z}}{\underline{Z}_{m} \| \underline{Z}+\underline{Z}_{1}}=\frac{1}{\operatorname{Re}+j \cdot \operatorname{Im}}$

$R e=1+\frac{Z_{1}}{Z} \cos \left(\varphi_{1}-\varphi_{z}\right)+\frac{Z_{1}}{Z_{m}} \cos \left(\varphi_{1}-\varphi_{m}\right)$

Im $=Z_{1}\left(\frac{1}{Z} \sin \left(\varphi_{1}-\varphi_{z}\right)+\frac{1}{Z_{m}} \sin \left(\varphi_{1}-\varphi_{m}\right)\right)$

$\underline{A}_{2}=\frac{\underline{v}_{2}}{\underline{v}_{0}}=\frac{\underline{Z}_{3}}{\underline{Z}_{3}+\underline{Z}_{2}} \cdot \frac{\underline{v}_{1}}{\underline{v}_{0}}=\frac{Z_{3}}{Z} e^{j\left(\varphi_{3}-\varphi_{z}\right)} \cdot \frac{\underline{v}_{1}}{\underline{v}_{0}}$

These expressions can easily be separated into real and imaginary part to obtain magnitude and phase. Figure 13 shows the exemplary amplitude response curves $\left|\underline{A}_{1}(\omega)\right|$ and $\left|\underline{A}_{2}(\omega)\right|$ for $k_{3}=0$ and $k_{3}=2 \times 10^{-5} \mathrm{Nm}^{-1}$ (in both cases $d_{3}=0$ ), which demonstrate the change in the amplitude response curves due to an external influence. It is obvious that
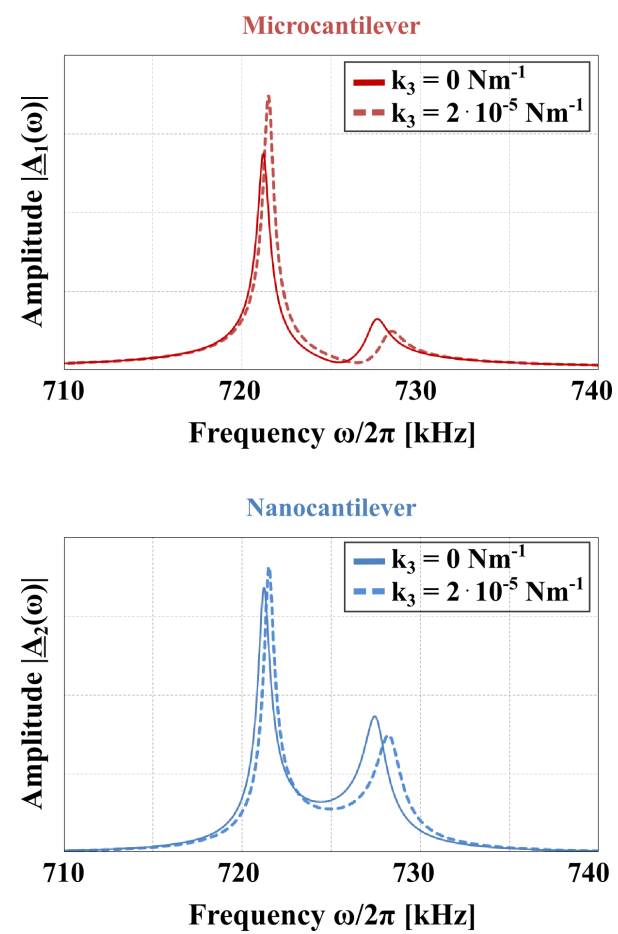

Figure 13. Amplitude curves for both cantilevers for the circuit model in Fig. 12 with and without an external interaction, represented by the spring $k_{3}$ and $d_{3}=0$.

the external interaction leads to a shift of the coupled system's resonance frequencies $\omega_{a, b}$ as well as a change of the resonance amplitudes.

In the following discussion we will only consider the additional spring $k_{3}$ as external interaction and neglect the damping element $d_{3}$, since this will enable a comparison between simulation results and exemplary measurements carried out with the sensor considered here.

\section{Effective sensor properties}

In the last section, the frequency shift for both resonance peaks of the coupled system caused by an additional spring $k_{3}$ is discussed and exemplary resonance curves are depicted in Fig. 13. However, the frequency shifts of both peaks are not equal: the one with the smaller amplitude exhibits a stronger frequency shift than the peak with the larger amplitude. This behaviour can be explained by a closer look at the properties of the coupled system. As already suggested by Torres et al. (2007), the properties of a coupled sensor system are a mixture of both subsystems' features. The differing frequency shifts of the resonance peaks furthermore indicate that the underlying sensor properties are different for each peak. Hence, the peak with the smaller amplitude is more strongly dominated by the nanocantilever than the peak with the higher amplitude. In the following, we introduce a single-harmonic-oscillator description for each of the reso- 

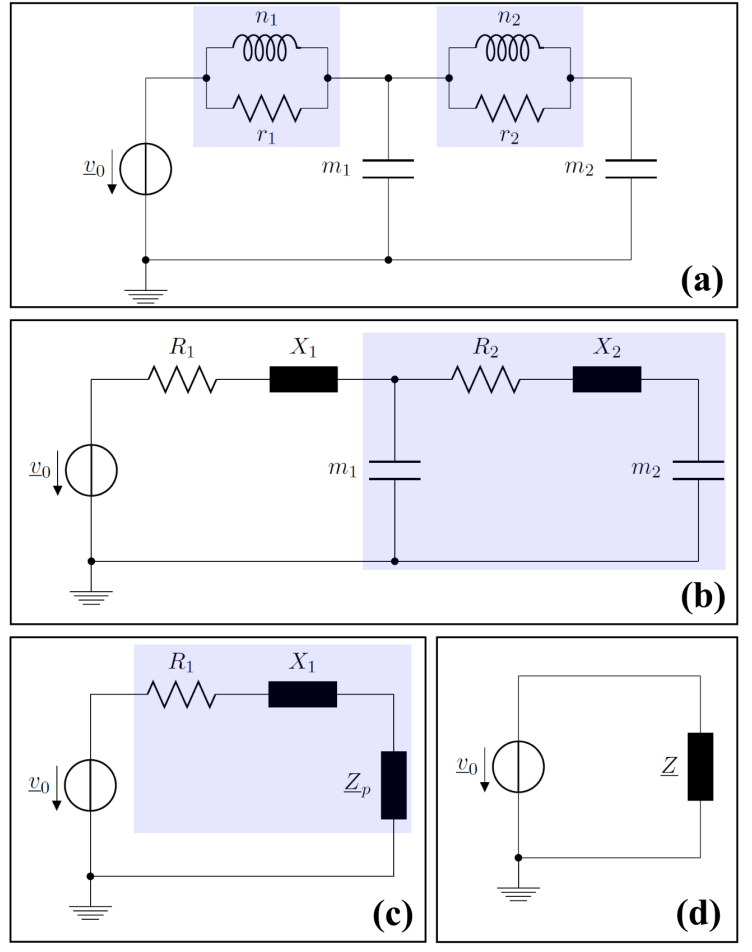

Figure 14. Transformation of the circuit model without external interaction (a) into a complex impedance $\underline{Z}$ : (b) conversion of $r-n$ parallel in series circuit, (c) combination of parallel branches into a complex impedance $\underline{Z}_{\mathrm{p}}$, (d) summation of $R_{1}, X_{1}$ and $\underline{Z}_{\mathrm{p}}$ to $\underline{Z}$.

nance peaks $\omega_{a}$ and $\omega_{b}$ to take the different behaviour into account and to obtain effective sensor properties describing the respective resonance peak. A corresponding derivation based on the circuit model is carried out, and the resulting effective sensor properties are verified by experimental data in Sect. 8, demonstrating the applicability of the approach. The knowledge of the effective sensor properties is not only a theoretical consideration but crucial for the practical evaluation of measured data. For example, in many applications it is important to know the effective spring constant describing each resonance peak in order to gain reliable information from measured data (Sader et al., 2012; Cleveland et al., 1993). Furthermore, gaining insight in the coupled sensor's effective properties allows for an optimal design process because the sensor's behaviour can be predicted and optimized more easily before actual fabrication.

The approach of determining the effective sensor properties is based on a series/parallel conversion of the circuit model in such a way that the coupled system is expressed as a complex impedance $\underline{Z}$ as depicted in Fig. 14d. This complex impedance is then compared to the total impedance $\underline{Z}_{\mathrm{p}}$ of the circuit model in Fig. 15b, which describes a single equivalent cantilever. In order to facilitate the necessary determination of real and imaginary part of this total impedance, the equivalent cantilever's circuit representation is transformed into a
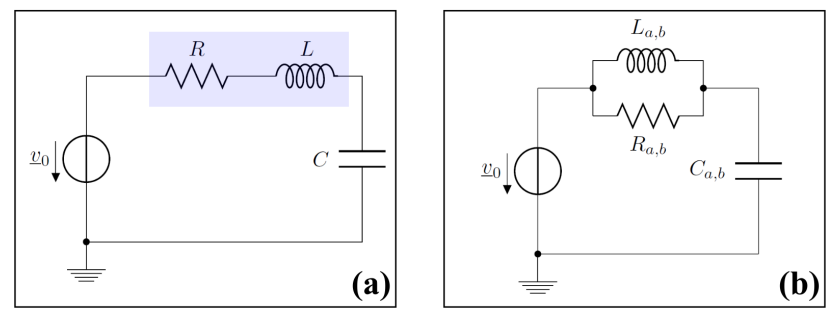

Figure 15. (a) Equivalent series resonance circuit for the circuit from Fig. 14 used to determine the effective properties of each resonance peak of the coupled system and (b) circuit model representing an effective cantilever.

series resonance circuit, with the total impedance $\underline{Z}_{r}$, by another series/parallel conversion (Fig. 15a). The elements of this circuit representation correspond to the mechanical elements as follows: resistor $\rightarrow$ inverse effective damping constant, inductance $\rightarrow$ inverse effective spring constant, capacitor $\rightarrow$ total effective mass. The transformation is only valid for one resonance frequency if numerical values are calculated; furthermore, it has to preserve the total impedance of the circuit as well as the phase relations for current and voltage (Weißgerber, 2009).

The transformation steps are depicted in Fig. 14, where first the parallel elements $r_{1}, n_{1}$ and $r_{2}, n_{2}$ are converted into series elements $R_{1}, X_{1}$ and $R_{2}, X_{2}$ respectively:

$$
\begin{aligned}
R_{1} & =\frac{r_{1}\left(\omega n_{1}\right)^{2}}{r_{1}^{2}+\left(\omega n_{1}\right)^{2}} ; & X_{1} & =\frac{n_{1} r_{1}^{2}}{r_{1}^{2}+\left(\omega n_{1}\right)^{2}} ; \\
R_{2} & =\frac{r_{2}\left(\omega n_{2}\right)^{2}}{r_{2}^{2}+\left(\omega n_{2}\right)^{2}} ; & X_{2} & =\frac{n_{2} r_{2}^{2}}{r_{2}^{2}+\left(\omega n_{2}\right)^{2}} .
\end{aligned}
$$

Further transformation of the resulting circuit leads to a total complex impedance $\underline{Z}$ given by

$$
\begin{aligned}
\underline{Z} & =\operatorname{Re}\{\underline{Z}\}+j \cdot \operatorname{Im}\{\underline{Z}\} \\
\operatorname{Re}\{\underline{Z}\} & =R_{1}+\frac{R_{2}\left(1-\omega m_{1} B+\omega m_{1} R_{2} B\right)}{\left(1-\omega m_{1} B\right)^{2}+\left(\omega m_{1} R_{2}\right)^{2}} \\
\operatorname{Im}\{\underline{Z}\} & =X_{1}+\frac{B\left(1-\omega m_{1} B\right)-\omega m_{1} R_{2}^{2}}{\left(1-\omega m_{1} B\right)^{2}+\left(\omega m_{1} R_{2}\right)^{2}} \\
B & =X_{2}-\frac{1}{\omega m_{2}} .
\end{aligned}
$$

This total impedance consists of a real and an imaginary part which can be compared to their respective counterparts of the series resonance circuit depicted in Fig. 15a consisting of the resistor $R$, the inductor $L$ and the capacitor $C$ with the total impedance $\underline{Z}_{r}$ :

$\underline{Z}_{r}=R+j\left(\omega L-\frac{1}{\omega C}\right)$.

The comparison yields $R$ and an infinite number of solutions for $L$ and $C$. Hence, in order to determine the correct solution, another boundary condition is necessary which 
can be obtained with the following considerations: for a single cantilever it is well known that a force gradient, which is small and sufficiently linear over a vibration period, can be translated into an interaction spring constant $k_{3}$ (Giessibl, 2003) as it is also considered in the circuit model in Fig. 3. This interaction spring constant will induce a frequency shift of the cantilever's eigenfrequency. In case of a small interaction, this frequency shift $\Delta \omega$ is related to the interaction spring constant $k_{3}$ and the cantilever's spring constant $k$ by the following expression (Giessibl, 2003):

$\frac{\Delta \omega}{\omega} \approx \frac{k_{3}}{2 k}$.

This formula can also be applied for the coupled system when each resonance peak is described as a single cantilever with effective properties. Hence, $k$ has to be substituted by $k_{\text {eff }}^{a}$ or $k_{\text {eff }}^{b}$, and if the additional spring $k_{3}$ is smaller than the smallest spring constant in the coupled system (Bhushan, 2010).

As the discussion in Sect. 6 showed, the amplitude curve of the coupled system with external interaction can be obtained from the circuit model. The determination of the resonance frequencies $\omega_{a}$ and $\omega_{b}$ of the coupled system with and without external interaction can either be done analytically or by means of a circuit analysis software. These calculations give the necessary frequency shifts $\Delta \omega_{a, b}$ for both resonance peaks for a known interaction spring constant $k_{3}$, as well as the resonance frequencies in case of neglected external interaction $\omega_{a, b}$. By rearranging Eq. (45), the effective spring constant $k_{\text {eff }}^{a, b}$ for each resonance peak can be determined. According to the analogies from Table $1, L=1 / k_{\text {eff. If the ca- }}$ pacitance $C$ (which corresponds to the effective mass) of the equivalent circuit is required, it can be determined by

$C=\frac{1}{\omega(\omega L-\operatorname{Im}\{\underline{Z}\})}$.

With these considerations, all elements of the series resonance circuit are defined. Hence, another series/parallel transformation of the resistor and inductor is carried out to obtain a circuit which is structurally equivalent to that of a cantilever, i.e. with inductor and resistor in parallel as shown in Fig. 15b. Both resonance peaks of the coupled system can be described by this equivalent cantilever with effective properties by employing either one of the resonance frequencies $\omega_{a}$ and $\omega_{b}$. The calculations have been carried out for the exemplary sensor, and the numerical values for the electrical as well as the mechanical elements are summarized in Table 5. As these values show, the effective parameters for the peaks differ due to a different influence of the subsystems. Please note that $C_{a, b}$ and subsequently $m_{a, b}$ have been calculated according to Eq. (46).

As the numerical values in the next section indicate, it is a valid approach to represent each of the coupled system's
Table 5. Calculated values for the equivalent circuit model for both resonance peaks of the coupled system in Fig. 15b based on the properties given in Table 2.

\begin{tabular}{ll}
\hline Electrical & Mechanical \\
\hline Left peak $f_{a}$ & \\
$L_{a}=35.71 \mathrm{H}$ & $k_{\text {eff }}^{a}=0.028 \mathrm{Nm}^{-1}$ \\
$C_{a}=1.37 \times 10^{-15} \mathrm{~F}$ & $m_{a}=1.37 \times 10^{-15} \mathrm{~kg}$ \\
$Q_{a}=1170$ & $Q_{a}=1170$ \\
$R_{a}=1.89 \times 10^{11} \Omega$ & $d_{a}=5.29 \times 10^{-12} \mathrm{~kg} \mathrm{~s}^{-1}$ \\
\hline Right peak $f_{b}$ & \\
$L_{b}=83.33 \mathrm{H}$ & $k_{\text {eff }}^{b}=0.012 \mathrm{Nm}^{-1}$ \\
$C_{b}=5.73 \times 10^{-16} \mathrm{~F}$ & $m_{b}=5.73 \times 10^{-16} \mathrm{~kg}$ \\
$Q_{b}=590$ & $Q_{b}=590$ \\
$R_{b}=2.25 \times 10^{11} \Omega$ & $d_{b}=4.45 \times 10^{-12} \mathrm{~kg} \mathrm{~s}^{-1}$ \\
\hline
\end{tabular}

resonance peaks by an equivalent single cantilever, hence a single harmonic oscillator. With this in mind, there is an alternative to calculate $m_{a, b}$ by employing the expression $\omega_{0}^{2}=k_{\text {eff }} / m_{\text {eff }}$ for the eigenfrequency $\omega_{0}$ of the harmonic oscillator. By rearranging it and using the effective spring constant $k_{\mathrm{eff}}^{a, b}$ and resonance frequency $\omega_{a, b}$ for the respective resonance peak, the effective mass of the equivalent harmonic oscillator representation can be obtained as well. However, this still requires the determination of the effective spring constant beforehand.

\section{Experimental verification of the circuit model}

In the experiment, the nanocantilever was made of an ironfilled carbon nanotube (FeCNT). Therefore, the external interaction was given by a magnetostatic interaction between the iron nanowire and a magnetic field introduced by a permanent magnet (Körner et al., 2016). The magnetic field can be estimated rather accurately by 2-D finite element simulation of the magnet with the software package FEMM (Meeker, 2015), leading to an interaction spring constant of $k_{3} \approx(-2 \pm 0.5) \times 10^{-5} \mathrm{Nm}^{-1}$ for a magnetic field of approximately $-400 \mathrm{mT}$ (Körner et al., 2016). Whereas the frequency shift of the sensor has been measured in different magnetic field strengths in the experiment, we will only choose one value as an example here. The complete measured data and further experimental details are discussed in another publication (Körner et al., 2016).

The value for $k_{3}$ has already been used for the exemplary calculation depicted in Fig. 13. Comparing this to the microcantilever's measured amplitude response curve depicted in Fig. 16, we find a good qualitative agreement. The values for the frequency shift induced by the external interaction are given in Table 6. Here we also find a good agreement, especially for the higher left resonance peak. The rather large difference of the frequency shift values for the smaller peak can be attributed to the measurement noise and the increased 
Table 6. Comparison of analytically derived and measured frequency shift values for an external interaction of $k_{3} \approx(-2 \pm 0.5) \times 10^{-5} \mathrm{Nm}^{-1}$.

\begin{tabular}{lrrr}
\hline & Measurement & Circuit model & Equivalent model \\
\hline Left peak $\Delta f_{a}$ & $(-278 \pm 20) \mathrm{Hz}$ & $(-257 \pm 51) \mathrm{Hz}$ & $(-258 \pm 52) \mathrm{Hz}$ \\
Right peak $\Delta f_{b}$ & $(-761 \pm 166) \mathrm{Hz}$ & $(-617 \pm 123) \mathrm{Hz}$ & $(-620 \pm 124) \mathrm{Hz}$ \\
\hline
\end{tabular}

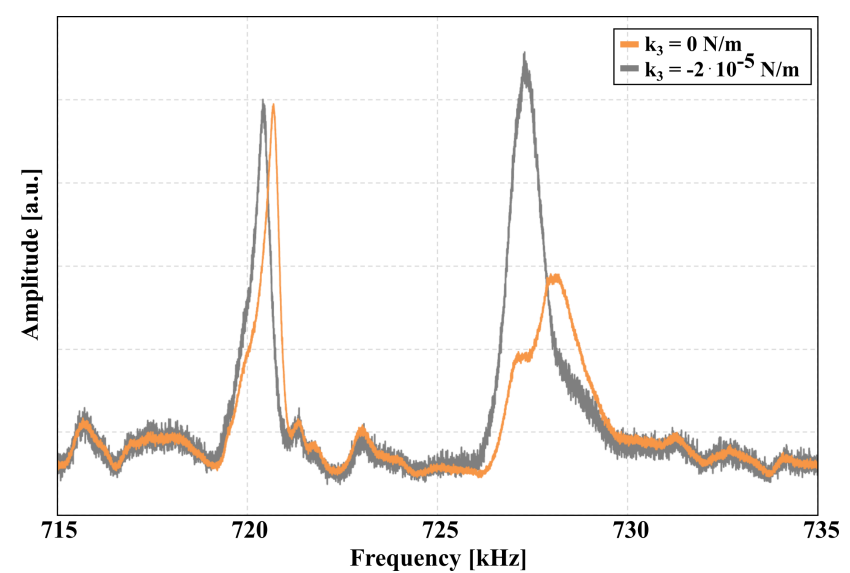

Figure 16. Amplitude response curve of the coupled system measured with laser deflection at the microcantilever under high vacuum $\left(\approx 10^{-5}\right.$ mbar $)$ and at room temperature.

width of this peak, making it difficult to determine its actual resonance frequency. In addition, one should always keep in mind that the values for the mechanical elements in the circuit model have also been obtained by measurements and, therefore, have an uncertainty. We assumed an uncertainty of $20 \%$ for the frequency shift values obtained with the circuit model, based on the uncertainty estimations for the interaction spring constant and the systems' spring constants (Clifford and Seah, 2005). From that we conclude that the circuit model is a valid description of the sensor's behaviour. Furthermore, we also applied the circuit model to other sensors in the same experiment and also found a good agreement between the measurement and simulation results.

As already mentioned above, we can also test the equivalent circuit model for each of the two resonance peaks $\omega_{a}$ and $\omega_{b}$ derived in Sect. 7 with the additional spring $k_{3}$. The results are given in Table 6 as well and agree with the measured values and the ones obtained with the complete circuit model within the margins of uncertainty for both peaks.

\section{Circuit model for a beam with shear and torsion}

In the previous sections we have seen that a simple coupled harmonic oscillator model describes the behaviour of a coresonantly coupled sensor very well. However, one has to keep in mind that this model is only valid for one resonance frequency. This is not a limitation for the case given, as only one flexural vibration mode of both beams is of interest.
However, the rather simple model does not take any shear deformation into account (Rossing and Fletcher, 2004), which may be of interest, e.g. if the sensor shall be used in a torsional vibration mode. To describe the one-side clamped beam in greater detail, a more complex model has to be used (Lenk et al., 2001). It consists of a translational and a rotatory part, and the beam is divided into smaller elements in order to linearize its behaviour. The chosen number of elements determines the number of vibration modes which can be considered with this model (Lenk et al., 2001).

Each element contains an inductor in the rotatory and a resistor and capacitor in the translational part. These two parts are connected via a current-controlled voltage source. For the set-up of two coupled cantilever beams, the corresponding circuit is given in Fig. 17. We chose a number of six elements to model each cantilever beam, which is sufficient since only the first resonance mode of the oscillating system is of interest here. Furthermore, the boundary conditions have to be defined, which are given by a fixed clamping on the left side, preventing any rotational movement. This would ideally be represented by a short circuit. For numerical stability of the circuit analysis software, a resistor $R_{1}$ with a very small resistance is used. Furthermore, the excitation applied to the microcantilever is modelled by a voltage source. The connection between the two cantilevers is assumed to be stiff and, therefore, direct connections are used. The nanocantilever's free end is not restrained in any case. Therefore, the resistor $R_{2}$ and inductor $L_{13}$ are used with very high values corresponding to an open circuit. We employed an inductor in the translational part in order to allow for modelling of the external interaction with the environment if desired. These boundary conditions are specific to our sensor set-up, but other boundary conditions can be found in Lenk et al. (2001) and Ballas et al. (2009).

Figure 18 depicts the Bode plots for both cantilevers, showing the first two vibrational modes for the microcantilever. A closer look at the first vibrational mode in Fig. 19 shows a very good agreement with the curves obtained with the circuit model for the coupled harmonic oscillator, indicating once more that this rather simple model can be used to obtain sufficient information on the coupled system's behaviour. 


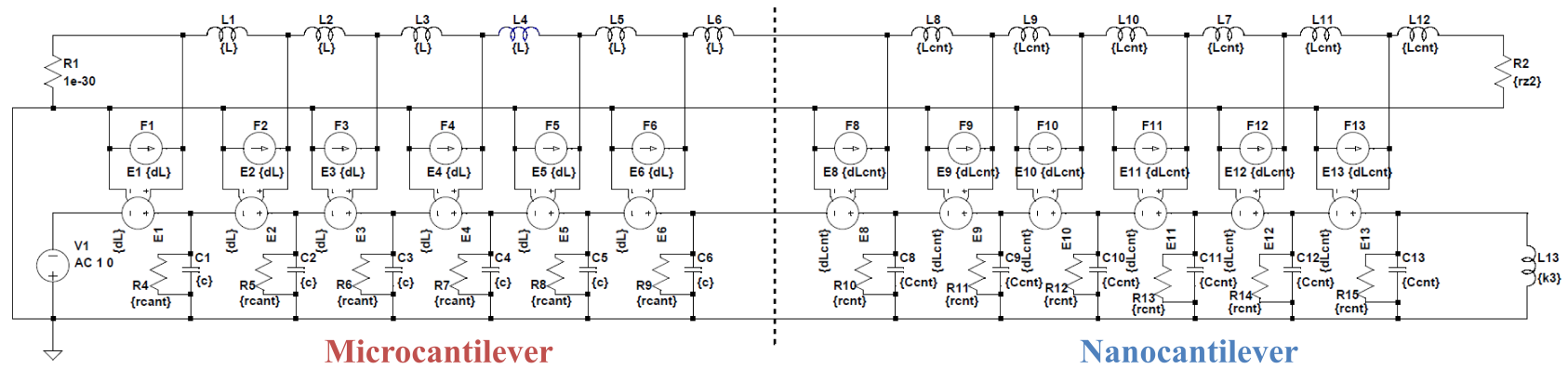

Figure 17. Circuit model of two coupled cantilever beams with translational and rotatory degrees of freedom of the movement.
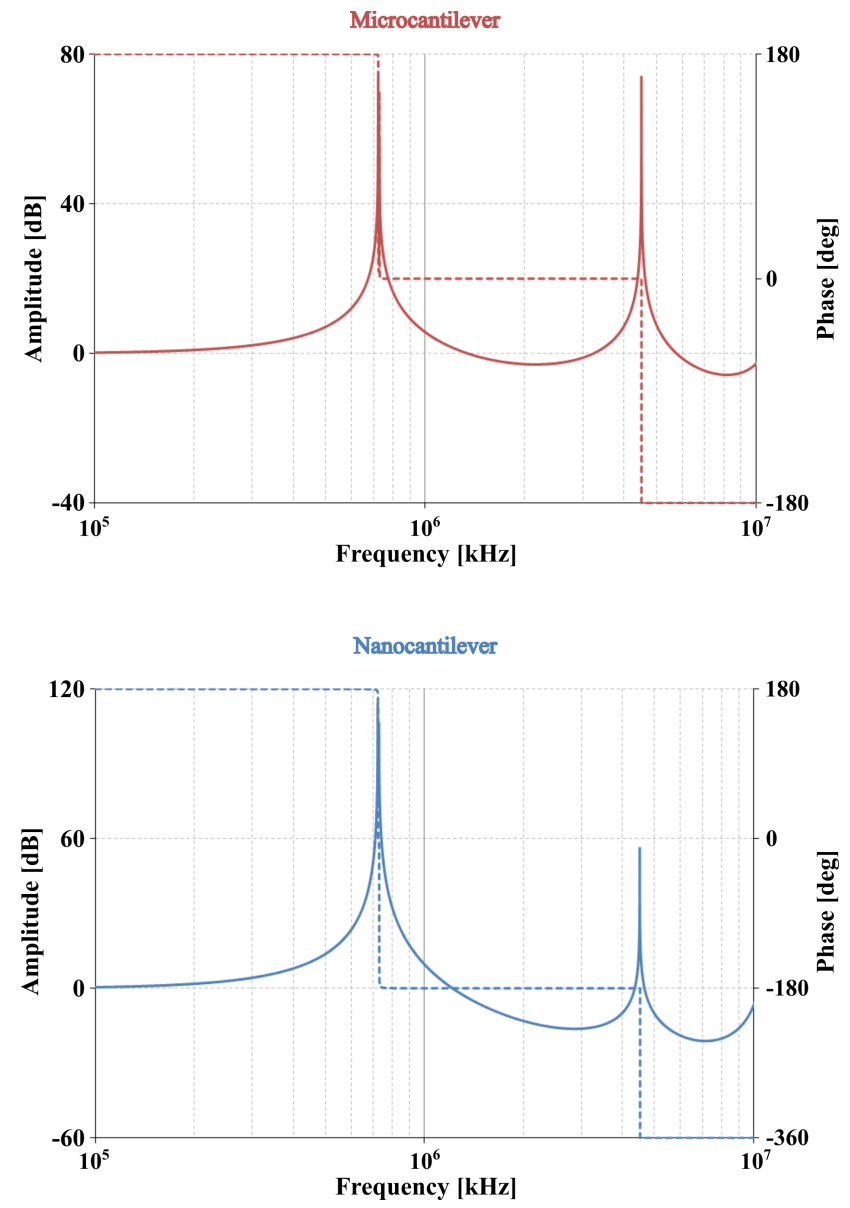

Figure 18. Bode plot for both cantilevers of the coupled system of Fig. 17 represented by a discrete beam model, depicting several resonance modes.

\section{Conclusions}

Application of electro-mechanical analogies is a commonly used tool to describe and analyse mechanical systems. We have applied the electric circuit model to our recently developed concept of a co-resonantly coupled sensor consisting of a micro- and a nanocantilever. The mechanical representation
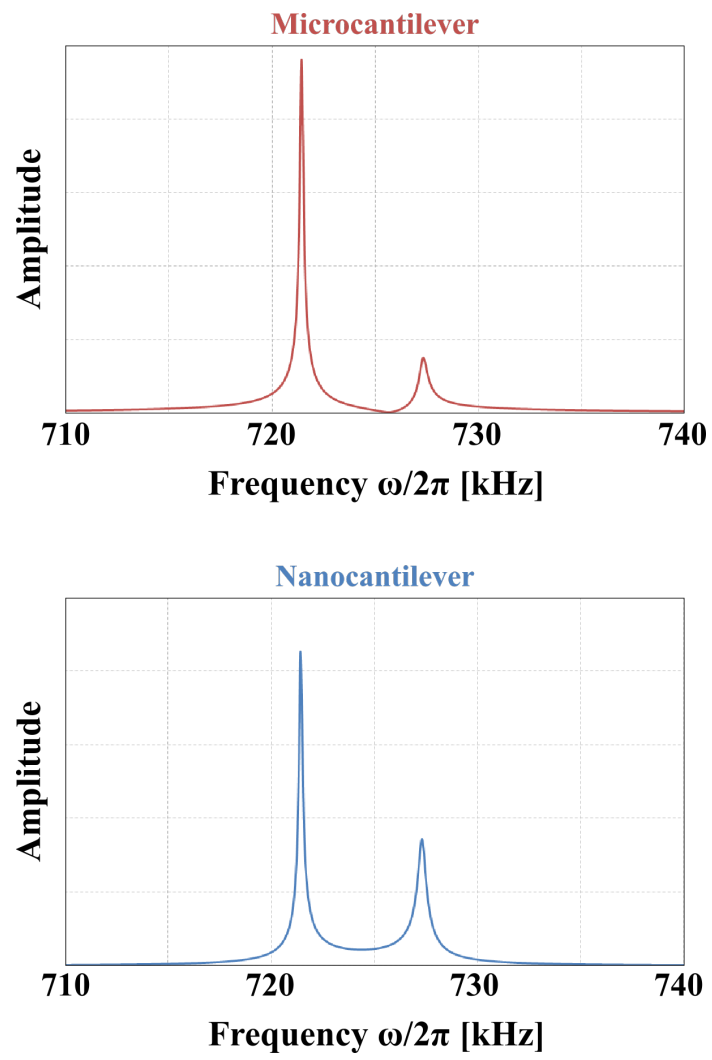

Figure 19. Amplitude curves for both cantilevers for the first bending mode obtained with the discrete beam model in Fig. 17.

of the sensor set-up can be considered as a simple coupled harmonic oscillator, which then has been transferred into an electric circuit. Analysis of the circuit can be performed completely analytically and with the software LTspice in order to gain profound understanding of the system's behaviour and to examine various features of the coupled system. This knowledge can be used to predict the behaviour of the sensor and help interpret and understand measurement results. Furthermore, the circuit model can also be employed for sensor design, allowing for determination of expected sensor characteristics such as the effective spring constant, which determines the sensitivity of the system. 
Additionally, the more complex model of a beam taking bending and shear deformation into account has been applied for the coupled sensor system. Generally, it has been shown that the simple coupled harmonic oscillator model describes the sensor's behaviour sufficiently well.

\section{Data availability}

The data presented in this paper is available on request from the corresponding author.

Acknowledgements. The authors acknowledge U. Marschner for helpful discussions regarding electro-mechanical networks. Furthermore, the authors acknowledge funding by the DFG (grant no. MU 1794/3-2).

Edited by: S. Schmid

Reviewed by: two anonymous referees

\section{References}

Axisa, F.: Modelling of Mechanical Systems - Discrete Systems, Kogan Page Science, London, 2nd Edn., 2004.

Ballas, R. G., Pfeifer, G., and Werthschützky, R.: Elektromechanische Systeme in Mikrotechnik und Mechatronik, Springer-Verlag, Berlin Heidelberg, 2nd Edn., 2009.

Belbasi, S., Foulaadvand, M. E., and Joe, Y. S.: Anti-resonance in a one-dimensional chain of driven coupled oscillators, Am. J. Phys., 82, 32-38, doi:10.1119/1.4827277, 2014.

Bhushan, B. (Ed.): Springer Handbook of Nanotechnology, Springer, London, New York, 3rd Edn., 2010.

Botman, A., Hesselberth, M., and Mulders, J. J. L.: Investigation of morphological changes in platinum-containing nanostructures created by electron-beam-induced deposition, J. Vac. Sci. Technol. B, 26, 2464, doi:10.1088/0957-4484/20/19/195301, 2008.

Bronstein, I. N., Semendjajew, K. A., Musiol, G., and Mühlig, H.: Taschenbuch der Mathematik, Verlag Harri Deutsch, Frankfurt am Main, 6th Edn., 2005.

Buchter, A., Nagel, J., Rüeffer, D., Xue, F., Weber, P. D., Kieler, O. F., Weinmann, T., Kohlmann, J., Zorin, A. B., Russo-Averchi, E., Huber, R., Berberich, P., Fontcuberta i Morral, A., Kemmler, M., Kleiner, R., Koelle, D., Grundler, D., and Poggio, M.: Reversal Mechanism of an Individual Ni Nanotube Simultaneously Studied by Torque and SQUID Magnetometry, Phys. Rev. Lett., 111, 067202, doi:10.1103/PhysRevLett.111.067202, 2013.

Cleveland, J. P., Manne, S., Bocek, D., and Hansma, P. K.: A nondestructive method for determining the spring constant of cantilevers for scanning force microscopy, Rev. Sci. Instrum., 64, 403-405, doi:10.1063/1.1144209, 1993.

Clifford, C. A. and Seah, M. P.: The determination of atomic force microscope cantilever spring constants via dimensional methods for nanomechanical analysis, Nanotechnology, 16, 1666, doi:10.1088/0957-4484/16/9/044, 2005.

Dorf, R. C. and Svoboda, J. A.: Introduction to electric circuits, John Wiley and Sons, New York, 8th Edn., 2010.
Firestone, F. A.: A new analogy between mechanical and electrical systems, J. Acoust. Soc. Am., 4, 249-267, doi:10.1121/1.1915605, 1933.

Giessibl, F. J.: Advances in atomic force microscopy, Rev. Mod. Phys., 75, 949, doi:10.1103/revmodphys.75.949, 2003.

Gil-Santos, E., Ramos, D., Martinez, J., Fernandez-Regulez, M., Garcia, R., San Paulo, A., Calleja, M., and Tamayo, J.: Nanomechanical mass sensing and stiffness spectrometry based on twodimensional vibrations of resonant nanowires, Nat. Nanotechnol., 5, 641, doi:10.1038/NNANO.2010.151, 2010.

Gysin, U., Rast, S., Aste, A., Speliotis, T., Werle, C., and Meyer, E.: Magnetic properties of nanomagnetic and biomagnetic systems analyzed using cantilever magnetometry, Nanotechnology, 22, 285715, doi:10.1088/0957-4484/22/28/285715, 2011.

Hähnle, W.: Die Darstellung elektromechanischer Gebilde durch rein elektrische Schaltbilder, Veröffentlichungen aus dem Siemens-Konzern, XI, 1-23, doi:10.1007/978-3-642-99668-9, 1931.

Joe, Y. S., Satanin, A. M., and Kim, C. S.: Classical analogy of Fano resonances, Phys. Scripta, 74, 259-266, doi:10.1088/00318949/74/2/020, 2006.

Klotter, K.: Die Analogien zwischen elektrischen und mechanischen Schwingern, Ingenieur Archiv, XVIII, 291-301, doi:10.1007/BF00538490, 1950.

Körner, J., Reiche, C. F., Büchner, B., Gerlach, G., and Mühl, T.: Application of a co-resonant sensor concept in cantilever magnetometry, 2015 IEEE 15th International Conference on Nanotechnology (IEEE-NANO), 1239-1242, doi:10.1109/NANO.2015.7388854, 2015.

Körner, J., Reiche, C. F., Büchner, B., Gerlach, G., and Mühl, T.: Signal enhancement in cantilever magnetometry based on a coresonantly coupled sensor, Beilstein J. Nanotechnol., accepted, 2016.

Lavenant, H., Naletov, V., Klein, O., de Loubens, G., Casado, L., and De Teresa, J. M.: Mechanical magnetometry of Cobalt nanospheres deposited by focused electron beam at the tip of ultra-soft cantilevers, Nanofabrication, 1, 65-73, doi:10.2478/nanofab-2014-0006, 2014.

Lenk, A., Pfeifer, G., and Werthschützky, R.: Elektromechanische Systeme, Springer-Verlag, Berlin Heidelberg, 1st Edn., 2001.

Li, X., Ono, T., Lin, R., and Esashi, M.: Resonance enhancement of micromachined resonators with strong mechanical-coupling between two degrees of freedom, Microelectron. Eng., 65, 1-12, doi:10.1016/S0167-9317(02)00595-6, 2003.

Meeker, D.: Finite Element Method Magnetics, Version 4.2, User's Manual, 2015.

Novotny, L.: Strong coupling, energy splitting, and level crossings: A classical perspective, Am. J. Phys., 78, 1199-1202, doi:10.1119/1.3471177, 2010.

Philippi, S., Weißker, U., Mühl, T., Leonhardt, A., and Büchner, B.: Room temperature magnetometry of an individual iron filled carbon nanotube acting as nanocantilever, J. Appl. Phys., 110, 084319, doi:10.1063/1.3651392, 2011.

Rast, S., Wattinger, C., Gysin, U., and Meyer, E.: Dynamics of damped cantilevers, Rev. Sci. Instrum., 71, 2772-2775, doi:10.1063/1.1150690, 2000.

Reiche, C. F., Körner, J., Büchner, B., and Mühl, T.: Introduction of a co-resonant detection concept for mechanical oscillation- 
based sensors, Nanotechnology, 26, 335501, doi:10.1088/09574484/26/33/335501, 2015a.

Reiche, C. F., Vock, S., Neu, V., Schultz, L., Büchner, B., and Mühl, T.: Bidirectional quantitative force gradient microscopy, New J. Phys., 17, 013014, doi:10.1088/1367-2630/17/1/013014, 2015 b.

Rossing, T. D. and Fletcher, N. H.: Principles of Vibration and Sound, Springer-Verlag, Berlin Heidelberg, 2nd Edn., 2004.

Rubbmark, J. R., Kash, M. M., Littman, M. G., and Kleppner, D.: Dynamical effects at avoided level crossings:A study of the Landau-Zener effect using Rydberg atoms, Phys. Rev. A, 23, 3107-3117, doi:10.1119/1.3471177, 1981.

Sader, J. E., Sanelli, J. A., Adamson, B. D., Monty, J. P., Wei, X., Crawford, S. A., Friend, J. R., Marusic, I., Mulvaney, P., and Bieske, E. J.: Spring constant calibration of atomic force microscope cantilevers of arbitrary shape, Rev. Sci. Instrum., 83, 103705, doi:10.1063/1.4757398, 2012.

Sökmen, U., Stranz, A., Waag, A., Ababneh, A., Seidel, H., Schmid, U., and Peiner, E.: Evaluation of resonating Si cantilevers sputter-deposited with AlN piezoelectric thin films for mass sensing applications, J. Micromech. Microeng., 20, 064007, doi:10.1088/0960-1317/20/6/064007, 2010.
Tipler, P. A. and Mosca, G. (Eds.): Physik für Wissenschaftler und Ingenieure, Springer, Berlin, Heidelberg, 7th Edn., 2015.

Torres, F., Abadal, G., Arcamone, J., Teva, J., Verd, J., Uranga, A., Lopez, J. L., Borrisé, X., Pérez-Murano, F., and Barniol, N.: Coupling Resonant Micro and Nanocantilevers to Improve Mass Responsivity by Detectability Product, Proceedings of the 14th International Conference on Solid-State Sensors, Actuators and Microsystems, p. 237, doi:10.1109/SENSOR.2007.4300113, 2007.

Vidal-Álvarez, G., Agustí, J., Torres, F., Abadal, G., Barniol, N., Llobert, J., Sansa, M., Fernández-Regúlez, M., PérezMurano, F., San Paulo, A., and Gottlieb, O.: Top-down silicon microcantilever with coupled bottom-up silicon nanowire for enhanced mass resolution, Nanotechnology, 26, 145502, doi:10.1088/0957-4484/26/14/145502, 2015.

Weißgerber, W.: Elektrotechnik für Ingenieure - Formelsammlung, Vieweg \& Teubner, Wiesbaden, 3rd Edn., 2009. 